\title{
1 Tempo and timing of ecological trait divergence in bird speciation
}

3 Jay P. McEntee*1,2 ${ }^{1,}$ Joseph A. Tobias ${ }^{3}$, Catherine Sheard ${ }^{4}$, \& J. Gordon Burleigh ${ }^{1}$

5 Affiliations:

$6{ }^{1}$ Biology Department, University of Florida, PO Box 118525, 220 Bartram Hall, Gainesville, FL 32611-8525, USA

$8{ }^{2}$ Ecology and Evolutionary Biology Department, University of Arizona, PO Box 210088,

9 Biological Sciences West Room 310, 1041 E. Lowell St., Tucson, Arizona 85721, USA

$10{ }^{3}$ Department of Life Sciences, Imperial College London, Silwood Park, Ascot, SL5 7PY, UK

$11{ }^{4}$ School of Biology, University of St. Andrews, Harold Mitchell Building, St Andrews Fife,

12 KY16 9TJ, United Kingdom

13 *corresponding author

15 Name and complete mailing address of the person to whom correspondence should be sent:

16 Jay McEntee, Biology Department, University of Florida, PO Box 118525, 220 Bartram Hall,

17 Gainesville, FL 32611-8525, USA Email: jaymcentee@,ufl.edu

\section{Abstract}

Organismal traits may evolve either gradually or in rapid pulses, but the relative importance of these modes in the generation of species differences is unclear. Additionally, while pulsed evolution is frequently assumed to be associated with speciation events, few studies have

23 explicitly examined how the tempo of trait divergence varies with respect to different

24 geographical phases of speciation, starting with geographic isolation and ending, in many cases,

25 with spatial overlap (sympatry). Here we address these issues by combining divergence time

26 estimates, trait measurements, and geographic range data for 952 avian sister species pairs

27 worldwide to examine the tempo and timing of trait divergence in recent speciation events. We

28 show that patterns of divergence in key ecological traits are not gradual, but instead appear to

29 follow a pattern of relative stasis interspersed with evolutionary pulses of varying magnitude. We

30 also find evidence that evolutionary pulses generally precede sympatry, and that major pulses

31 leading to greater trait disparity are associated with sympatry. These findings suggest that early 
32 pulses of trait divergence promote subsequent transitions to sympatry, rather than occurring after

33 sympatry has been established. Incorporating models with evolutionary pulses of varying

34 magnitude into speciation theory may explain why some species pairs achieve rapid sympatry

35 whereas others undergo prolonged geographical exclusion.

Speciation in vertebrates may proceed over long and variable periods ${ }^{1-6}$. From onset to completion, the process is often viewed as a cycle with three stages, beginning with geographic isolation (allopatry), followed by secondary contact initiated at range edges, and finally

41 prolonged spatial coexistence in overlapping geographical ranges (sympatry; see Fig. 1) ${ }^{1,5-7}$.

42 The process does not always proceed to the second or third stages, but the establishment of

43 sympatry has been viewed by some as the only way to be certain that speciation is complete ${ }^{2}$. In

44 some forms of this model, here termed "geographic speciation," sympatry can be delayed after

45 secondary contact has been made, either by competitive interactions ${ }^{8}$ or incomplete reproductive

46 isolation ${ }^{5}$. Thus sympatry may only occur when species traits are sufficiently divergent ${ }^{9}$, while

47 recently diverged species with similar traits fail to overlap, and instead exhibit abutting ranges

48 (termed parapatry). However, while the pattern of increased trait divergence in sympatric versus 49 non-sympatric sister lineages is widespread among animal taxa ${ }^{10,11}$, the timing and geographical 50 context of trait divergence is difficult to determine. In particular, trait divergence could arise 51 primarily by the accumulation of differences either prior to sympatry ${ }^{12}$ or after sympatry is 52 established (e.g. via character displacement) ${ }^{13}$.

53 The tempo and mode of ecological trait divergence during the geographic speciation

54 cycle are also debated ${ }^{14,15}$, with some studies describing divergence as slow or gradual 55 throughout the process ${ }^{4,12}$, while others describe abrupt, pulse-like changes occurring against a 56 background of $\operatorname{stasis}^{16}$. Pulsed changes could occur either early, in allopatry or parapatry ${ }^{17,18}$, or

57 later, in sympatry ${ }^{9,13}$ (Fig. 1). It is further possible that ecological (local) adaptation in

58 allopatry/parapatry, followed by subsequent species interactions in sympatry provide the context

59 for multiple pulses of trait divergence over the course of geographic speciation cycles ${ }^{17}$.

60 Assessing the relative contributions of gradual versus pulse-and-stasis evolution to

61 species differences involves challenges associated with temporal scale ${ }^{19}$ and analytical

62 approach $^{14}$. On short timescales, microevolutionary studies indicate that stasis generally

63 dominates $^{20}$, with abrupt pulses of divergence occasionally detected ${ }^{21}$. It is unclear whether these 
64 pulses ultimately contribute to species-level differences or simply represent brief departures of 65 trait values from longer-term static, or gradually changing, means ${ }^{10,14,20,22}$. Fossil time series, 66 which allow analyses of complex mixtures of modes over timescales relevant to geographic 67 speciation ( $10^{4}$ to $10^{7}$ years), show evidence for gradual change and stasis within lineages ${ }^{23,24}$. 68 Taxonomic issues and patchy geographic sampling of the fossil record, however, limit the extent 69 to which the fossil record can be used to assess phenotypic divergence as a component of the 70 geographic speciation cycle ${ }^{14}$.

These challenges have led to an increased use of phylogenetic approaches to assess the 72 tempo and mode of phenotypic change associated with speciation. These methods generally use

73 data from extant species to test for correlations between trait evolution and speciation ${ }^{25}$ or to

74 partition trait evolution between cladogenetic and anagenetic change ${ }^{26}$. These approaches

75 suggest that pulse-like speciational (cladogenetic) processes may contribute to trait divergence.

76 However, such analyses are often conducted across large clades, potentially overlooking the role

77 of extinction in generating observed patterns in extant diversity ${ }^{27-29}$. Moreover, the assumption in 78 most phylogenetic approaches that evolutionary pulses occur only at speciation ${ }^{15}$, which is 79 modelled as a single instantaneous event ${ }^{30,31}$, limits how much these approaches can tell us about 80 the roles of pulsed and gradual evolution over the extended course of geographic speciation 81 cycles.

82 Disentangling alternative divergence pathways at different stages of speciation is a key

83 step to understand general patterns of trait evolution and predict the fate of nascent species when 84 changing environments redistribute geographic ranges ${ }^{2,18,32}$. For example, minor trait divergence 85 during the allopatric phase of speciation may be ephemeral if gene pools merge during secondary 86 contact, whereas greater levels of divergence may lead to reproductive isolation and ultimately 87 sympatry. This latter possibility could accentuate patterns of pulsed evolution in phylogenies and 88 the fossil record, even when divergence itself is gradual ${ }^{18,32}$. Framed by these uncertainties, our 89 understanding of the rates and timing of trait divergence in vertebrate speciation remains highly 90 incomplete, not least because the data required are often patchy for large samples of species. In 91 particular, for most large taxonomic groups the information on ecological trait divergence and 92 geographic distributions is not sufficiently resolved to explore divergence pathways in the 93 context of geographical phases of speciation. 
To address these issues, we examine phenotypic divergence, geographic relationships,

95 and divergence times among 952 pairs of avian sister species. By restricting the analysis to sister

96 species, we explicitly focus on ecological trait divergence associated with relatively recent

97 speciation events, which reduces the impact of species extinction on our inferences relative to

98 clade-wide approaches ${ }^{27,33}$. For each pair of species, we estimated trait divergence and

99 determined geographical relationships using a geospatial database of $\sim 178$ million species

100 observation records ${ }^{34,35}$ and a set of standard range polygons. Combining detailed phenotypic,

101 temporal and spatial information, we consider (1) whether pulse-and-stasis or gradual evolution

102 predominate in ecological trait divergence over the extended duration of bird speciation, (2)

103 whether divergence in ecological traits is associated with sympatry establishment, and (3) how

104 the estimated timing of divergence relates to the geographic phases of speciation.

105

\section{Results}

107 Using estimated divergence times for species pairs generated from phylogenetic data ${ }^{36-38}$, along

108 with estimates of trait disparity for each pair of species, we fitted a set of evolutionary

109 divergence models ${ }^{22}$ designed to span microevolutionary and macroevolutionary processes. We

110 assessed relative support for four stochastic models: a Brownian motion ("gradual") divergence

111 model, a "single pulse" divergence model, a "multiple pulse" divergence model, and a time-

112 independent ("white noise") divergence model. The first three models (gradual, single pulse,

113 multiple pulse) incorporate an additional white noise component to represent bounded evolution

114 processes at shorter timescales and to help to account for measurement error. In the single and

115 multiple pulse models, the pulses are modeled as instantaneous jumps in trait values, which are

116 representative of brief periods of high-rate directional evolution ${ }^{22}$ as expected in rapid evolution

117 toward a new adaptive optimum ${ }^{39}$ and not as saltational jumps. We interpret the single pulse

118 model as a "pulse-and-stasis" model, as the bounds of the bounded evolution component are

119 narrow $^{40}$ (Supplementary Tables 1 through 4). For two important ecological traits -- body mass

120 and beak morphology (see Methods) -- the single pulse model has strong support relative to the

121 other three models. Sensitivity analyses (Supplementary Tables $1-4$ ) indicated that these results

122 were robust to an alternate approach to phylogenetic reconstruction and divergence time

123 estimation $^{38}$. 
The difference in support between the single pulse and gradual models is consistent with

125 phylogenetic studies that have found evidence for a contribution of speciational pulses in the 126 accumulation of phenotypic diversity ${ }^{26}: \Delta$ AIC between the single pulse and Brownian motion

127 models is 899 for body mass, $\triangle$ AICs between the single pulse and Brownian motion models are 128 808, 920, and 967 for culmen length, beak depth, and beak width, respectively (see Methods and 129 Supplementary Tables 1 through 4). Our analyses suggest, however, that such pulses need not be 130 completely coincident with speciation, i.e. as reconstructed as instantaneous events (nodes) on 131 phylogenetic trees. Instead, such pulses may take place at some point in the course of a 132 protracted geographic speciation process ${ }^{30}$, as indicated by non-zero estimated waiting times to 133 pulses.

134 To estimate the timing of these divergence pulses, we used the rate parameter in the 135 single pulse model preferred in our analyses. For body mass, the expected waiting time to a pulse 136 was $\sim 670,000$ years ( $95 \% \mathrm{CI}$ from likelihood profile: 275,000 years to $1.13 \mathrm{My}$; see also Figs. 2 137 and 3, Supplementary Table 1). The expected waiting times to a pulse in beak morphology 138 divergence in single pulse models were $\sim 440,000$ years for culmen length (95\% CI: 200,000 to 139930,000 years), 360,000 years for beak depth (95\% CI: 100,000 to 720,000 years), and $140 \sim 420,000$ years for beak width (95\% CI: 130,000 to 820,000 years; see Supplementary Tables 2 $1414)$.

142 To compare the accumulation of trait divergence with transitions from allopatry to 143 sympatry, we calculated and visualized the cumulative distribution function (CDF) for 144 undergoing a pulse of divergence over time (Fig. 3, see also Supplementary Figure 1). The pulse 145 magnitudes in the trait divergence models are variable (specifically they are drawn from a 146 normal distribution with a mean of 0 ), such that we only expect large-magnitude pulses for a 147 small fraction of species pairs. Most species pairs in this model accumulate modest trait 148 divergence. Under this model, however, large-magnitude divergence is possible not only for 149 older species pairs, but also for younger species pairs.

150 To compare our estimates of pulse timing and trait divergence accumulation with the 151 progression to secondary contact (inclusive of both parapatry and sympatry) and sympatry, we 152 obtained information on contact from a geospatial database of $\sim 178$ million species observation 153 records $^{34,35}$ and on sympatry from standard geographical range polygons ${ }^{41}$ (Fig. 4). Comparing 154 the inferred timescales of trait divergence and sympatry suggests that pulses of trait divergence 
155 largely precede the establishment of sympatry (Fig. 3), which occur predominantly on the order 156 of $10^{6}$ years. This result is contingent on the assumption that transitions out of sympatry are

157 infrequent ${ }^{7}$. The relative timescales of inferred trait divergence pulses and sympatry

158 establishment (Fig. 3) suggest that many divergence pulses occur in the absence of sympatric

159 interactions that could cause character displacement ${ }^{13}$. By contrast, we found that secondary

160 contact occurs early enough and frequently enough among species pairs that the signature of

161 initial allopatry is absent in our analyses of secondary contact and divergence time

162 (Supplementary Figure 3; Supplementary Information: Timing of secondary contact and

163 sympatry), leaving open the possibility that trait divergence often follows secondary contact, or

164 that parapatric speciation (without an initial allopatric stage) occurs with regularity among birds

165 (see below, Methods and Supplementary Information).

166 Pulses of ecological trait divergence theoretically reduce competition and reproductive

167 interference among incipient species ${ }^{17}$, potentially overcoming constraints on sympatry

168 establishment ${ }^{2,7}$. The possibility that divergence by pulse-and-stasis evolution is widespread

169 during speciation raises the question of whether divergence pulses are consequential for

170 transitions through the geographic speciation process. Thus we tested whether variation in body

171 mass and beak morphology predicted which species pairs are parapatric (with abutting

172 distributions) or sympatric (with overlapping distributions). Focusing on all species pairs in

173 secondary contact ( $n=440$, see Methods), and accounting for the effects of divergence time,

174 dispersal ability, and latitude, we found evidence that sympatry is associated with greater

175 divergence in ecological traits (Fig. 4, Supplementary Tables 8 - 9). In different analyses, either

176 body mass divergence or aspects of beak morphology divergence had more support as predictors

177 of sympatry (Supplementary Tables $8-9$ ). Relationships between increased probability of

178 sympatry and ecological trait divergence were present across sensitivity analyses accounting for

179 a different approach to phylogenetic reconstruction and divergence time estimation

180 (Supplementary Tables 12 - 13), and an alternate threshold for delineating sympatry versus

181 parapatry (Supplementary Tables $14-15$ ). These results are largely in agreement with previous

182 studies showing that the transition from secondary contact to sympatry is associated with

183 divergence in body mass and/or beak morphology ${ }^{11,12}$.

184 The hand-wing index, a morphological proxy of dispersal ability ${ }^{42,43}$, was also a

185 consistent predictor of sympatry for species pairs in contact across analyses (Fig. 5, 
186 Supplementary Information). This effect could be observed if sympatric populations tend to be

187 sinks supported by non-sympatric sources: species with elevated dispersal capacity may maintain

188 sympatric sink populations whereas those with weak dispersal may fail to. Additionally,

189 increased dispersal capacity may be associated with elevated founder population sizes or

190 densities during colonization of the sister species' range. These elevated founder population sizes

191 may facilitate colonization within the ranges of sister species by surmounting the challenges of

192 population establishment at low density or number ${ }^{44,45}$.

193 Lastly, we inferred that contact between members of species pairs often occurs early (i.e.

194 within the first two million years) in the geographic speciation cycle (Supplementary Fig. 3),

195 contrary to models of geographic speciation that specify that long periods of allopatry are

196 necessary for speciation ${ }^{2}$. This suggests a potentially wider role for parapatric speciation

197 (speciation with no stage $a$ in Fig. 1) ${ }^{4}$ than is often considered in bird speciation ${ }^{46}$. In parapatric

198 speciation, divergence pulses prior to sympatry would necessarily occur despite contact, and thus

199 the potential for gene flow, between incipient species ${ }^{17}$. However, we also found that observed

200 patterns of contact and divergence time among bird sister species could be explained by

201 speciation models requiring some period of allopatry at the outset, but allowing for rapid rates of

202 transition to secondary contact (i.e. speciation with reduced duration of stage $a$ in Fig. 1;

203 Supplementary Figs. 9 - 10, Supplementary Information). Stochastic modeling of species ranges

204 indicated than an approximate minimum rate of transition to secondary contact of 0.3 transitions

205 per million years is sufficient to explain the pattern of contact among sister pairs. That is, a $30 \%$

206 probability of a species pair coming into contact within a million years is sufficient to yield a

207 combined set of divergence times for species pair contact approximating the empirical

208 observations. Thus, trait divergence pulses may occur during periods of either allopatry or

209 parapatry, with cases of both likely widespread. Our evidence for early contact during speciation

210 suggests that selection (e.g. local adaptation, immigrant inviability ${ }^{47}$ or other immigrant

211 disadvantages, and/or selection against intermediate phenotypes) frequently plays a role in

212 driving or maintaining divergence in the early stages of speciation.

213

214

215

216

\section{Discussion}

A prevailing view on bird speciation is that ecological trait divergence sufficient to

217 enable sympatry requires long periods of allopatry ${ }^{9,12}$. Gradual evolution models, such as the 
218 random walk models used in many phylogenetic comparative methods for continuous traits, are 219 consistent with this prevailing view ${ }^{7,15}$. However, gradual divergence models inadequately

220 account for strong divergence in young species pairs, unless they also incorporate brief bursts of 221 faster gradual divergence ${ }^{48,49}$, similar to pulses. Even the Early Burst model ${ }^{50}$, used to represent 222 the explosively rapid trait divergence dynamics in extant adaptive radiations ${ }^{28}$, poorly accounts

223 for highly divergent recent species pairs because it predicts comparatively slow evolutionary 224 rates at the tips of phylogenetic trees. Thus, while widely used gradual models may adequately 225 explain avian trait evolution at deeper macroevolutionary scales (as for comparing genera or 226 families $^{28}$ ), our findings indicate that patterns of divergence among sister pairs are better 227 captured by models incorporating pulsed divergence amid periods of stasis. In particular, the 228 single pulse model we test here receives relatively strong support, and can help to explain the range of geographic speciation outcomes observed in nature, which includes many "old" species 230 pairs that are highly similar in ecological traits ${ }^{9}$ but also instances of abrupt ecological trait 231 divergence in young species pairs ${ }^{10,17,51,52}$.

232 The signal of trait divergence pulses during the geographic speciation cycle raises the 233 question of how such pulses may take place. We suggest that pulses are likely to result from the 234 intermittent discovery, by populations or lineages, of unoccupied adaptive peaks, as is expected 235 in niche-filling models of diversification ${ }^{6,10}$, or more generally from adaptive evolution on 236 heterogeneous fitness landscapes ${ }^{14,53}$. Unoccupied adaptive peaks may become occupied, via 237 pulses of adaptive directional evolution, during or immediately following range expansion ${ }^{16,54}$, 238 for instance immediately following colonization of novel environments. Rapid phenotypic 239 divergence may also result from local adaptation along environmental gradients, with or without 240 gene flow ${ }^{55}$. In such contexts, signals of pulsed divergence could plausibly arise from a 241 combination of local (clinal) adaptation and the subsequent extinction of phenotypically 242 intermediate populations ${ }^{16,18,56}$. A key shared aspect of all these scenarios is that evolution on 243 adaptive landscapes allows for pulse-and-stasis modes of divergence ${ }^{14}$. Our data may also be 244 consistent with a role for character displacement ${ }^{57}$ driving pulsed divergence in some instances.

245 Comparing the timescales of trait divergence and sympatry establishment (Fig. 3), however, 246 suggests that divergence pulses more frequently result from processes not requiring sympatry 247 than processes that require sympatry. 
Our finding that greater divergence in ecological traits is positively associated with the

249 probability of sympatry accords well with what has been found previously in many other studies 250 of vertebrates ${ }^{58,59}$, including birds ${ }^{11,12}$, using a variety of different methodological approaches.

251 The focus of such studies is commonly character displacement ${ }^{11,58,60}$, yet this association can

252 also be explained by the spatial sorting of species ranges by competition ${ }^{12,61}$ or because these

253 trait differences may be associated with stronger reproductive isolation ${ }^{5,62}$. Tobias et al. ${ }^{12}$ found

254 that the association of greater divergence in size and beak morphology of sympatric versus non-

255 sympatric ovenbird species could be explained by a third factor, time since divergence, which

256 they interpreted as evidence for spatial sorting of ecologically diverged lineages. Our results

257 provide indirect support for this sorting hypothesis in a sample of species pairs that spans the

258 diversity of birds, in that the timescale of ecological trait divergence under the single pulse

259 model is faster than the relatively slow accumulation of sympatry sister species pairs (Fig. 3) ${ }^{62}$.

260 These findings suggest that pulses of trait divergence promote subsequent transitions to

261 sympatry, rather than such pulses primarily occurring through character displacement after

262 sympatry has been established. This interpretation is contingent on the assumption that the

263 movement of species ranges over time results in low transition rates of sympatry to non-

264 sympatry in sister pairs, i.e. that the curve in Fig. 3 is mostly representative of the initial

265 achievement of secondary sympatry and not of an equilibrium between relatively even transition 266 rates into and out of sympatry ${ }^{7,63}$.

267 In combination, our results may help to resolve the longstanding question of why only

268 some nascent species survive over evolutionary time. One of the major threats to young lineages

269 is fusion through swamping gene flow upon contact ${ }^{2}$. We have demonstrated that this risk is

270 widespread among nascent bird species because the lag time to secondary contact is generally

271 short (Supplementary Figs. 3 and 6 - 8), supporting the view that gene flow routinely becomes

272 possible early in the speciation process. In particular, species pairs with minimally divergent

273 phenotypes may incur increased hybridization rates or increased hybrid fitness relative to more

274 diverged pairs, potentially leading to the extinction of one member of the species pair.

275 Importantly, though, our findings also suggest that species pairs undergoing major early pulses

276 of ecological trait divergence are more likely to rapidly transition to sympatry, escaping both

277 fusion and mutual exclusion, and possibly extending their lifespan as independent lineages ${ }^{56,64-}$

$278 \quad{ }^{66}$. Elevated rates of extinction in less-divergent young lineages may increase the signature of 
279 large early pulses in datasets compiled from extant species, such as that presented here. Thus,

280 differential extinction coupled with pulses of early trait divergence may play a critical role in

281 explaining broad-scale patterns in the longevity, macroevolutionary diversity, and geographical

282 distribution of species.

\section{METHODS}

285 Sister pairs We selected all $(\mathrm{n}=2,076)$ pairs of avian sister species (i.e. each other's closest

286 relatives) from the maximum likelihood topology from Burleigh et al.' $\mathrm{s}^{36}$ avian supermatrix

287 phylogenetic analysis (hereafter "Burleigh tree"), which contains 6,714 species of the $\sim 10,500$

288 bird species in the world. The inclusion of pairs of non-sister lineages would not invalidate our

289 analyses, but for the purposes of interpretation we excluded pairs that may not represent true

290 sister species because one taxon may have a closer relative. Specifically, we excluded 763 pairs

291 belonging to genera with $<75 \%$ species-level sampling, and another 62 pairs that were deemed

292 unlikely to be true sister species based on either molecular evidence from other studies or

293 taxonomic problems. For most analyses, we further removed 299 species pairs for which we

294 could not adequately score the presence or absence of contact (Supplementary Dataset 6).

295 Different analyses used different subsets of sister pairs, depending on data availability and

296 quality (see Supplementary Datasets 1 - 6). In using the sister pair data set to make inferences

297 about the speciation process, we leveraged the broad variation in divergence times and stages of

298 the geographic speciation cycle represented among sister pairs, including pairs that are in the

299 early stages of divergence.

300 Divergence times Divergence time estimates were obtained from an ultrametric tree inferred

301 from the maximum likelihood tree from Burleigh et al. ${ }^{36}$ using penalized likelihood analysis

302 implemented in $\mathrm{r} 8 \mathrm{~s}^{37,67}$. The r8s analysis used 20 fossil calibrations and constrained the root of

303 the tree to a maximum age of 110 mya. However, applying a maximum age constraint to the root

304 node had little effect on estimates of sister pair divergence times (Supplementary Fig. 5). A list

305 of the fossil calibrations (Supplementary Information Dataset 7, see also ${ }^{68}$ ) and a command

306 block for the r8s analyses are available in the Supplementary Information. We performed

307 sensitivity analyses using alternate sets of divergence time estimates, both from bootstrap

308 analysis of the Burleigh tree, and from an independent phylogenetic and dating analysis ${ }^{38}$ (see

309 Supplementary Information). 
310 Ecological trait measurements Body mass Divergence in body size may be a strong contributor

311 to ecological divergence, potentially reducing interspecific competition ${ }^{10,69}$ or reproductive

312 interference ${ }^{5}$. We compiled data on body mass (a proxy for body size) for species pairs from

313 updated global datasets ${ }^{70-72}$. When multiple body mass values were reported, we took the mean;

314 when male and female body masses were reported separately, we calculated an average of the

315 two sex-specific means. We estimated body mass disparity as the difference between species in

316 natural log of mean body mass. This estimate is a unitless measurement representing

317 proportional change in body mass ${ }^{22}$.

318 Beak morphology Species with similar body mass may partition niches according to diet. Thus,

319 to quantify differences in foraging ecology among sister species, we collected three beak

320 measurements (culmen length, beak depth, beak width) associated with food item selection and

321 manipulation ${ }^{7,73,74}$. Culmen length was measured as the distance from the distal part of the

322 nostril to the beak tip. Beak depth and beak width were both measured at the distal edge of the

323 nostril. All beak measurements were made on wild birds or museum specimens using calipers, to

324 the nearest $0.1 \mathrm{~mm}$ ( $\mathrm{n} \geq 4$ individuals sampled per species, $\geq 2$ males and $\geq 2$ females, where

325 possible; see Supplementary Information for further details about the beak morphology data set).

326 For evolutionary mode analyses, we estimated proportional differences between species'

327 phenotypes by taking the difference of the natural log of the mean body mass of each species ${ }^{22}$.

328 To calculate multivariate species differences, we first fit separate phylogenetic generalized linear

329 regressions of the log species means of each of the beak measurements on species log mean body

330 mass (with Ornstein-Uhlenbeck errors ${ }^{75}$, see Supplementary Information section 5: Predictors of

331 contact and sympatry). We then calculated the Mahalanobis distance between the three beak

332 measurement residuals from the phylogenetic generalized linear regressions for each species pair

333 to estimate multivariate divergence in beak morphology.

334 Dispersal Highly vagile taxa with greater dispersal capacities should undergo faster range

335 expansions, potentially leading to earlier secondary contact in nascent species ${ }^{43}$. This can be

336 associated with faster transition rates to sympatry ${ }^{63}$, but when secondary contact is very early, it

337 may also slow or reverse the speciation process by promoting gene flow, leading to merged gene

338 pools rather than continued divergence ${ }^{2}$. Because of the importance of dispersal in geographic

339 speciation models, we assessed how dispersal capacity influences transitions from allopatry to

340 secondary contact, and from secondary contact to sympatry, respectively. As it is difficult to 
341 measure dispersal capacity directly, we instead used the hand-wing index (HWI), an index of

342 wing shape related to the aspect ratio of the wing ${ }^{43}$ and a proxy for flight performance and

343 dispersal capacity in birds ${ }^{42,43,63,76}$. Using measurements (to the nearest $0.5 \mathrm{~mm}$ ) taken from

344 wild birds and museum specimens, we calculated this index as

345

346

$$
H W I=100 \times \frac{W L-S L}{W L}
$$

347 where WL (wing length) is the length of the closed wing from carpal joint to wing tip, and SL

348 (secondary length) is the distance from the carpal joint to the tip of the first secondary feather. As 349 a secondary index of dispersal, we also used range maps ${ }^{41}$ to assess migratory behavior. If either

350 member of a pair was illustrated as migratory to any degree, the species pair was scored as

351 migratory.

\section{Geographical phases of speciation}

354 Secondary contact We examined evidence for contact in species pairs using $~ 178$ million bird

355 species observation records stored in the eBird observational record database ${ }^{34,77}$. We

356 downloaded the ebird Basic Dataset (EBD_relOct-2013) from the eBird website

357 (http://ebird.org). For a given species pair, contact was defined by evidence for the co-occurrence

358 of both species on the same day at the same reported locality. Evidence that such co-occurrence

359 takes place was interpreted as evidence for contact between species ranges. We wrote a Perl

360 script that, for all sister species pairs, identified all instances when both species were listed at the

361 exact same latitude and longitude on the same day. However, the spatial extent of eBird

362 checklists varies when it is reported (Supplementary Figure 4), such that some apparent co-

363 occurrence records might come from checklists made for large areas or long transects.

364 Furthermore, some co-occurrence records may result from human-aided introductions to areas

365 extrinsic to native ranges or from ambiguity in different taxonomic treatments. To address these

366 issues, we compared our contact scoring with what would result from contact scoring from range

367 maps $^{41}$ and examined eBird records where there was any discrepancy (see additional details in

368 Supplementary Information: General supplementary methods). These data quality checks

369 resulted in the re-scoring of 15 species pairs (from having contact to having no contact) and the

370 exclusion of an additional 29 species pairs from the analysis where contact could not be scored

371 with confidence (Supplementary Dataset 6). 

member of a species pair is migratory, we scored breeding contact for such species pairs

374 specifically by examining whether co-occurrence took place in the breeding range and breeding 375 season, using breeding range maps ${ }^{41}$ and breeding phenology descriptions ${ }^{70}$ respectively. To

376 qualify as evidence of breeding contact, species co-occurrences in species pairs with any

377 migratory behavior had to be reported on the same day and in the same locality during the known

378 breeding seasons of both species ${ }^{70}$ and within the known breeding range of at least one of the

379 two species ${ }^{41}$. For species pairs without known migratory behavior, we assumed evidence of 380 contact was indicative of breeding contact (see also Supplementary Information).

381 Because co-occurrence is unlikely to be reported for species with very few observations,

382 we excluded sister pairs where at least one species had fewer than 10 eBird sightings reported.

383 Our contact scores likely underestimate the true extent of contact among species pairs, as even

384 after this filtering process, the minimum number of observations strongly predicts the probability

385 of species pair contact in our data set (GLM with the log of the minimum observations as sole

386 predictor: coefficient estimate $=3.8 \times 10^{-4} \pm 8.5 \times 10^{-5} \mathrm{SE}$; Supplementary Table 5).

387 Consequently, we conducted sensitivity analyses adopting an alternate minimum threshold, 20 or

38850 instead of 10, for observations (Supplementary Table 5 and Supplementary Fig. 8). We also

389 checked observational evidence for contact, discounting co-occurrence cases likely attributable

390 to anthropogenic introductions, and excluding cases potentially based on misidentifications or

391 taxonomic confusion (see included and excluded species pairs in Supplementary Datasets 1 and

392 6).

393 Sympatry To examine the transition from contact to broad range overlap (sympatry), we

394 calculated percent breeding range overlap from geographic range polygons ${ }^{41}$ with a custom $\mathrm{R}$

395 script, using the R libraries rgdal, rgeos, maptools, and raster. A small subset of species pairs (n

$396=17$ of 440 species pairs) could not be scored using our automated routine, and their range

397 overlap was estimated visually. Species pairs with a range overlap $>20 \%$ of the smaller range ${ }^{63}$,

$3987^{78,79}$ were scored as sympatric, while those with contact but with $\leq 20 \%$ range overlap were 399 scored as parapatric (having abutting ranges) ${ }^{63}$. This range overlap threshold may seem high 400 based on the definition of parapatry as the circumstance where ranges abut but are separate ${ }^{80}$. 401 Range maps are coarse-grained, however, such that ranges that do not substantially overlap in 402 reality appear to have overlap in range maps. As this $20 \%$ threshold, like any threshold, was 
403 somewhat arbitrary, we performed additional analyses using an overlap of $>10 \%$ scored as

404 sympatric (Supplementary Tables 14-15).

\section{Analyses}

\section{Tempo of ecological trait divergence}

407 To examine the tempo and timing of trait divergence, we evaluated the relative support for four 408 divergence models across the set of species pairs for which body mass or beak morphology data 409 were available ( $n=869$ species pairs for body mass, $n=945$ species pairs for beak morphology).

410 For all four traits, we defined disparities as the between-species difference in the $\log \operatorname{mean}^{22}$. We

411 fit one model of time-independent bounded evolution, as well as three different models that

412 comprise a bounded evolution component on shorter timescales and one of three additional

413 components for longer timescales ${ }^{22}$. These longer-timescale components are a gradual evolution

414 model (Brownian motion) and two forms of pulsed divergence: a single pulse model where a

415 single instantaneous displacement occurs following a waiting time sampled from an exponential

416 distribution and a multiple pulse model where the expected number of displacements for a given

417 divergence time is determined by a Poisson process. We examined relative support for these

418 models using AIC from likelihood calculations performed in R. We calculated confidence

419 intervals for the Poisson rate parameter $\lambda$, the inverse of which is taken as the expected waiting

420 time to a pulse, using likelihood profiling. To generate likelihood profiles, we calculated the

421 likelihood of single pulse models with a series of fixed $\lambda$ values at increments of 0.01 , over an

422 interval containing the maximum likelihood parameter estimate. We assume the log likelihood

423 ratio is chi-square distributed, and define the $95 \%$ confidence interval for $\lambda$ as the interval for

424 which the likelihood is within 1.92 units of the maximum likelihood model. To visualize the

425 cumulative probability of incurring a pulse for Fig. 3, we used the equation for the cumulative

426 distribution function for an exponential distribution, $1-e^{-\lambda t}$.

427 Secondary contact and sympatry We examined the probability of contact versus non-contact, and 428 parapatry versus sympatry, using GLM with binomial error distributions, implemented in $\mathrm{R}^{81}$. In 429 analyses of contact and breeding contact for sister pairs, we began by predicting the probability 430 of contact, with divergence time as the only predictor (Extended Data Figs 3a-b and 7). We 431 subsequently performed a model generation and selection routine (using the genetic algorithm of $432 \mathrm{R}$ package glmulti ${ }^{82}$, see Supplementary Information) to examine which among a set of 433 phenotypic measures best predict contact or sympatry while accounting for the effects of three 
434 variables that may influence the timing of transitions from allopatry to sympatry: divergence 435 time $^{12}$, latitude ${ }^{5}$, and dispersal ability ${ }^{63}$. The predictors of primary interest were between-species 436 disparity in two traits implicated in ecological and reproductive isolation: body mass ${ }^{83}$ and beak 437 morphology ${ }^{84}$. We incorporated disparity in beak morphology (i) as the Mahalanobis distance 438 between species residuals from phylogenetic linear regressions of beak measurements on body 439 mass (see above), or (ii) by including each of the three residuals (from phylogenetic linear 440 regressions of culmen length, beak width, and beak depth on body mass) as predictors. We used 441 disparities in mean body mass and beak morphology as predictors in these analyses instead of 442 sex-specific disparities as (i) many individuals in these datasets are not sexed, (ii) overall sexual 443 size dimorphism is minor, and (iii) species interactions involve both males and females, such that 444 the mean of these measurements is likely relevant.

445 To account for differences among sister species pairs in dispersal ability, we also 446 included the average log hand-wing index ${ }^{43}$ and migratory status of the sister pair as predictors.

447 We further included divergence time and midpoint latitude (average of the two median 448 observational latitudes for each species from $\mathrm{eBird}^{77}$ ). Our model generation routine permitted 449 all pairwise interactions between predictors to enter the model, under the constraint that all 450 models were marginal. We report support for all predictors entering the set of contact models 451 with $\triangle \mathrm{AIC}<2$ (Supplementary Tables 6 - 7). All continuous variables were scaled and centered, 452 such that estimated slope magnitudes for individual variables are meaningful in relation to one 453 another.

454 For GLM examining the probability of sympatry versus parapatry, we first limited the 455 sister species data set to those pairs with breeding contact. This restriction focuses the analysis 456 on taxa that have the opportunity to interact to some degree in the breeding season ${ }^{85}$. The 457 response variable in GLM is the geographic configuration: parapatric (interacting but without 458 substantial range overlap) versus sympatric (having substantial range overlap: $>20 \%$ of the 459 smaller range in the analyses presented in the main text). We again used a genetic algorithm (see 460 Supplementary Information) to generate model variants and performed model selection using the 461 R package glmulti ${ }^{82}$.

462 To assess the sensitivity of our results to uncertainty in phylogenetic inference and 463 divergence time estimates, we repeated all GLM analyses using mean divergence times for our 464 species pairs from 100 samples of the pseudo-posterior distribution of trees from an alternative 
465 Bayesian species-level phylogenetic analysis ${ }^{38}$ (hereafter, the "Jetz tree"; Supplementary Tables

4669 -12). For analyses examining the probability of contact (and breeding contact) with divergence

467 time, we performed additional sensitivity analyses using divergence time estimates from 100

468 bootstraps of the Burleigh tree and for each of the 10,000 pseudo-posterior samples from the Jetz 469 tree (Supplementary Fig. 7).

471 Simulations of range dynamics To aid in the interpretation of our GLM predicting local co472 occurrence, we performed stochastic range dynamic simulations ${ }^{63}$. We used these simulations to 473 place an approximate lower bound on the rate of secondary contact establishment from an 474 initially allopatric configuration. To perform this estimation, we simulated the establishment of 475 secondary contact using a simple model ${ }^{7,63}$, in which sister pairs can be in one of two states: co476 occurring and not co-occurring. We simulated transitions into and out of contact over a set of 477 possible rates from 0.1 to 0.8 per million years, in which the forward rate (rate of transition from 478 isolation to contact, $\sigma$ ) is always greater than or equal to the reverse rate (rate of transition out of 479 contact, $\varepsilon$ ). The forward and reverse rates are constant ${ }^{63}$, and the variation in rates among species 480 arises only from stochasticity. Reverse rates were simulated at $0.005,0.01,0.05,0.1,0.2$, and 0.5 481 times each of the forward rates. We present the maximum intercept calculated across all reverse 482 rates $(\varepsilon)$ for each simulated forward rate $(\sigma)$ (Extended Data Figs 5, 12). To calculate the 483 approximate percentage of species pairs coming into secondary contact by given points in time 484 following divergence (100,000 years, 1 million years), we simulated range dynamics with $\sigma=$ 4850.3 , and $\varepsilon=0.15$ (corresponding to the minimal $\sigma$ that yielded intercept $>0.434$, and the value of $486 \varepsilon$ that yielded the highest intercept for $\sigma=0.3$ ).

487 Code availability Computer code for analyses not found in the Supplementary Information can 488 be obtained from the authors upon request.

\section{References}

492 1. Mayr, E. in Systematics and the Origin of Species (Columbia University Press, 1942).

493 2. Mayr, E. in Animal Species and Evolution (Belknap Press of Harvard University Press, 494 Cambridge, Massachusetts, 1963).

495 3. Mayr, E. \& Diamond, J. M. in The birds of northern Melanesia: speciation, ecology \& 496 biogeography (Oxford University Press on Demand, 2001).

497 4. Price, T. in Speciation in Birds (Roberts and Company, Greenwood, Village, Colorado, 2008). 
5. Weir, J. T. \& Price, T. D. Limits to speciation inferred from times to secondary sympatry and ages of hybridizing species along a latitudinal gradient. Am. Nat. 177, $462-469$ (2011).

500 6. Price, T. D. et al. Niche filling slows the diversification of Himalayan songbirds. Nature 509, $501222-225$ (2014).

502 7. Pigot, A. L. \& Tobias, J. A. Species interactions constrain geographic range expansion over evolutionary time. Ecol. Lett. 16, 330-338 (2013). 8. MacArthur, R. \& Levins, R. The limiting similarity, convergence, and divergence of coexisting species. Am. Nat., 101, 377-385 (1967).

506

507 9. Rundell, R. J. \& Price, T. D. Adaptive radiation, nonadaptive radiation, ecological speciation and nonecological speciation. Trends Ecol. Evol. 24, 394-399 (2009). 10. Schluter, D. in The Ecology of Adaptive Radiation (Oxford University Press, Oxford, UK, 2000). 11. Bothwell, E., Montgomerie, R., Lougheed, S. C. \& Martin, P. R. Closely related species of birds differ more in body size when their ranges overlap-in warm, but not cool, climates. Evolution 69, 1701-12 (2015).

12. Tobias, J. A. et al. Species coexistence and the dynamics of phenotypic evolution in adaptive radiation. Nature 506, 359-363 (2014).

13. Pfennig, D. W. \& Pfennig, K. S. Character displacement and the origins of diversity. Am. Nat. 176 Suppl 1, S26-44 (2010).

14. Hunt, G. \& Rabosky, D. L. Phenotypic evolution in fossil species: pattern and process. Annu. Rev. Earth Planet. Sci. 42, 421-441 (2014).

15. Pennell, M. W., Harmon, L. J. \& Uyeda, J. C. Is there room for punctuated equilibrium in macroevolution? Trends Ecol. Evol. 29, 23-32 (2014).

16. Mayr, E. in Evolution as a process. (eds. Huxley, J., Hardy, A.C., \& Ford, E.B.) 157-180 (Allen \& Unwin, 1954).

17. Rundle, H. D. \& Nosil, P. Ecological speciation. Ecol. Lett. 8, 336-352 (2005).

18. Futuyma, D. J. On the role of species in anagenesis. Am. Nat. 130, 465-473 (1987).

19. Gingerich, P. Rates of evolution: effects of time and temporal scaling. Science 222, 159-162 (1983).

20. Estes, S. \& Arnold, S. J. Resolving the paradox of stasis: Models with stabilizing selection explain evolutionary divergence on all timescales. Am. Nat. 169, 227-244 (2007).

21. Boag, P. T. \& Grant, P. R. Intense natural selection in a population of Darwin's finches (Geospizinae) in the Galapagos. Science, 214, 82-85 (1981).

22. Uyeda, J. C., Hansen, T. F., Arnold, S. J. \& Pienaar, J. The million-year wait for macroevolutionary bursts. Proc. Natl. Acad. Sci. U. S. A. 108, 15908-15913 (2011). 23. Hunt, G., Hopkins, M. J. \& Lidgard, S. Simple versus complex models of trait evolution and stasis as a response to environmental change. Proc. Natl. Acad. Sci. U. S. A. 112, 4885-4890 (2015).

24. Prothero, D. R. et al. Size and shape stasis in late Pleistocene mammals and birds from Rancho La Brea during the Last Glacial-Interglacial cycle. Quat. Sci. Rev. 56, 1-10 (2012). 25. Rabosky, D. L. \& Adams, D. C. Rates of morphological evolution are correlated with species richness in salamanders. Evolution 66, 1807-1818 (2012).

26. Bokma, F. Time, species, and separating their effects on trait variance in clades. Syst. Biol. 59, 602-607 (2010). 
544

28. Cooney, C. R. et al. Mega-evolutionary dynamics of the adaptive radiation of birds. Nature 542, 344-347 (2017).

29. Marshall, C. R. Five palaeobiological laws needed to understand the evolution of the living biota. Nat. Ecol. Evol. 1, 165-017-0165 (2017).

30. Etienne, R. S., Morlon, H. \& Lambert, A. Estimating the duration of speciation from phylogenies. Evolution 68, 2430-2440 (2014).

31. Kuchta, S. R. \& Wake, D. B. Wherefore and whither the ting species? Copeia 104, 189-201 (2016).

32. Futuyma, D. J. in Macroevolution 29-85 (Springer, 2015).

33. Weir, J. T. \& Wheatcroft, D. A latitudinal gradient in rates of evolution of avian syllable diversity and song length. Proc. Roy. Soc. B 278, 1713-1720 (2011).

34. Sullivan, B. L. et al. eBird: A citizen-based bird observation network in the biological sciences. Biol. Conserv. 142, 2282-2292 (2009).

35. Sullivan, B. L. et al. The eBird enterprise: An integrated approach to development and application of citizen science. Biol. Conserv. 169, 31-40 (2014).

36. Burleigh, J. G., Kimball, R. T. \& Braun, E. L. Building the avian tree of life using a largescale, sparse supermatrix. Mol. Phylogenet. Evol. 84, 53-63 (2015).

37. Baiser, B., Valle, D., Zelazny, Z. \& Burleigh, J. G. Non random patterns of invasion and extinction reduce phylogenetic diversity in island bird assemblages. Ecography (2017).

38. Jetz, W., Thomas, G. H., Joy, J. B., Hartmann, K. \& Mooers, A. O. The global diversity of birds in space and time. Nature 491, 444-448 (2012).

39. Hunt, G., Bell, M. A. \& Travis, M. P. Evolution toward a new adaptive optimum: phenotypic evolution in a fossil stickleback lineage. Evolution 62, 700-710 (2008).

40. Arnold, S. J. Phenotypic evolution: the ongoing synthesis (American Society of Naturalists address). Am. Nat. 183, 729-746 (2014).

41. BirdLife International and NatureServe. in Bird species distribution maps of the world (BirdLife International and Natureserve, Cambridge, UK and Arlington, USA, 2014).

42. Dawideit, B. A., Phillimore, A. B., Laube, I., Leisler, B. \& Böhning Gaese, K.

Ecomorphological predictors of natal dispersal distances in birds. J. Anim. Ecol. 78, 388-395 (2009).

43. Claramunt, S., Derryberry, E. P., Remsen, J. V.Jr. \& Brumfield, R. T. High dispersal ability inhibits speciation in a continental radiation of passerine birds. Proc. Roy. Soc. B 279, 1567-1574 (2012).

44. Stephens, P. A., Sutherland, W. J. \& Freckleton, R. P. What is the Allee effect? Oikos 87, 185-190 (1999).

45. Case, T. J., Holt, R. D., McPeek, M. A. \& Keitt, T. H. The community context of species' borders: ecological and evolutionary perspectives. Oikos 108, 28-46 (2005).

46. Phillimore, A. B. et al. Sympatric speciation in birds is rare: Insights from range data and simulations. Am. Nat. 171, 646-657 (2008).

47. Nosil, P. in Ecological speciation (Oxford University Press, 2012).

48. Landis, M. J., Schraiber, J. G. \& Liang, M. Phylogenetic analysis using Levy processes: finding jumps in the evolution of continuous traits. Syst. Biol. 62, 193-204 (2013).

49. Landis, M. J. \& Schraiber, J. G. Pulsed evolution shaped modern vertebrate body sizes. Proc. Natl. Acad. Sci. U. S. A. (2017).

50. Harmon, L. J. et al. Early Bursts of Body Size and Shape Evolution are Rare in Comparative Data. Evolution 64, 2385-2396 (2010). 
51. Benkman, C. W. Divergent selection drives the adaptive radiation of crossbills. Evolution 57, 591 1176-1181 (2003).

592 52. Lamichhaney, S. et al. Evolution of Darwin's finches and their beaks revealed by genome sequencing. Nature 518, 371-375 (2015). 53. Gavrilets, S. Evolution and speciation on holey adaptive landscapes. Trends Ecol. Evol. 12, 307-312 (1997). 54. Friis, G., Aleixandre, P., Rodríguez Estrella, R., Navarro Sigüenza, A. G. \& Milá, B. Rapid postglacial diversification and long-term stasis within the songbird genus Junco: phylogeographic and phylogenomic evidence. Mol. Ecol. 25, 6175-6195 (2016). 55. Endler, J. A. in Geographic variation, speciation, and clines (Vol. 10) (Princeton University Press, 1977).

602

603

604 56. Futuyma, D. J. Evolutionary constraint and ecological consequences. Evolution 64, 18651884 (2010).

57. Brown, W. L. \& Wilson, E. O. Character displacement. Syst. Zool. 5, 49-64 (1956).

58. Dayan, T. \& Simberloff, D. Ecological and community wide character displacement: the next generation. Ecol. Lett. 8, 875-894 (2005).

59. Jonathan Davies, T., Meiri, S., Barraclough, T. G. \& Gittleman, J. L. Species co existence and character divergence across carnivores. Ecol. Lett. 10, 146-152 (2007).

60. Drury, J.P., Grether, G.F., Garland, T., \& Morlon, Hélène. An assessment of phylogenetic

611

612

613

614

615

616

617

618 tools for analyzing the interplay between interspecific interactions and phenotypic evolution. Sys. Biol. doi: https://doi.org/10.1093/sysbio/syx079. (2017).

61. Roughgarden, J. Competition and theory in community ecology. Am. Nat. 122, 583-601 (1983).

62. Hudson, E. J. \& Price, T. D. Pervasive reinforcement and the role of sexual selection in biological speciation. J. Hered. 105, 821-833 (2014).

63. Pigot, A. L. \& Tobias, J. A. Dispersal and the transition to sympatry in vertebrates. Proc. Roy. Soc. B 282, 20141929 (2015).

64. Rosenzweig, M. L. in Species diversity in space and time (Cambridge University Press, 1995).

65. Rosenblum, E. B. et al. Goldilocks meets Santa Rosalia: an ephemeral speciation model explains patterns of diversification across time scales. J. Evol. Biol. 39, 255-261 (2012).

66. Dynesius, M. \& Jansson, R. Persistence of within species lineages: a neglected control of speciation rates. Evolution 68, 923-934 (2014).

67. Sanderson, M. R8s: Inferring absolute rates of molecular evolution and divergence times in the absence of a molecular clock. Bioinformatics 19, 301-302 (2003).

68. Ksepka, D. \& Clarke, J. Phylogenetically vetted and stratigraphically constrained fossil calibrations within Aves. Palaeontol. Electron. 18, 1-25 (2015).

69. Wilson, D. S. The adequacy of body size as a niche difference. Am. Nat. 109, 769-784 (1975).

70. Handbook of the Birds of the World Alive (eds. Del Hoyo, J., Elliott, A., Sargatal, J.,

634 72. Dunning, J.B. Body Masses of Birds of the World.

635 https://ag.purdue.edu/fnr/Documents/WeightBookUpdate.pdf. (2016). 
73. Schoener, T. W. The evolution of bill size differences among sympatric congeneric species of birds. Evolution 19, 189-213 (1965).

638 74. Miles, D. B. \& Ricklefs, R. E. The correlation between ecology and morphology in deciduous forest passerine birds. Ecology 65, 1629-1640 (1984).

640 75. Ho, L. \& Ane, C. A linear-time algorithm for Gaussian and non-Gaussian trait evolution 641 models. Syst. Biol. 63, 397-408 (2014).

642 76. Stoddard, M. C. et al. Avian egg shape: Form, function, and evolution. Science 356, 1249-

6431254 (2017).

644 77. Sullivan, B. L. et al. The eBird enterprise: An integrated approach to development and 645 application of citizen science. Biol. Conserv. 169, 31-40 (2014).

646 78. Chesser, R. T. \& Zink, R. M. Modes of speciation in birds: a test of Lynch's method. 647 Evolution 48, 490-497 (1994).

648 79. Barraclough, T. G. \& Vogler, A. P. Detecting the geographical pattern of speciation from species-level phylogenies. Am. Nat. 155, 419-434 (2000). 80. Bull, C. Ecology of Parapatric Distributions. Annu. Rev. Ecol. Syst. 22, 19-36 (1991). 81. R Core Team. R: A language and environment for statistical computing. (2012).

653 82. Calcagno, V. \& de Mazancourt, C. glmulti: an R package for easy automated model selection with (generalized) linear models. Journal of Statistical Software 34, 1-29 (2010).

83. Yasukawa, K. Male quality and female choice of mate in the red-winged blackbird (Agelaius phoeniceus). Ecology 62, 922-929 (1981).

657 1-28 (1997).

658 85. Lovette, I. J. \& Hochachka, W. M. Simultaneous effects of phylogenetic niche conservatism and competition on avian community structure. Ecology 87, S14-S28 (2006).

\section{Acknowledgements}

662 We are grateful to numerous data collectors who contributed to eBird, GenBank, and the CRC

663 bird body mass data set (see Supplementary Information). We also thank Nico Alioravainen, Ed

664 Braun, Samuel Jones, Rebecca Kimball, Dan Ksepka, Monte Neate-Clegg, Alex Pigot, Aaron

665 Ragsdale and Gleb Zhelezov for data collection and technical assistance. We thank three

666 anonymous reviewers and Vera Domingues for constructive comments that improved the

667 manuscript. This work was supported by the National Science Foundation (DEB-1208428 to

668 J.G.B.), the Natural Environment Research Council (NE/I028068/1 to J.A.T.), and the Oxford

669 Clarendon Fund and US-UK Fulbright Commission (to C.S.).

671 Author contributions J.G.B. and J.P.M. conceived the study; J.G.B, J.P.M. and J.A.T. designed

672 the conceptual framework and analyses; J.G.B. performed dating analyses and assembled

673 phylogenetic, occurrence, and body mass information; J.A.T. and C.S. provided morphometric

674 data; J.P.M. integrated data sets, and designed and performed statistical analyses with significant 
675 input from J.G.B., J.A.T., and C.S.; J.P.M. and C.S. produced figures and tables; J.P.M. wrote

676 the manuscript, with significant input from all authors.

678 Author Information Reprints and permissions information is available at

679 www.nature.com/reprints. The authors declare no competing financial interests. Readers are

680 welcome to comment on the online version of this article at www.nature.com/nature.

681 Correspondence and requests for materials should be addressed to jaymcentee@ufl.edu.

Data Availability

684 We provide the data sets used to perform the analyses in this manuscript in a Microsoft Excel file 685 (.xlsx) in the Supplementary Information.

\section{Figures and figure legends}

691 Bird speciation typically involves a sequence of geographical states, starting with an allopatric 692 phase (a), followed by secondary contact initiated at range edges (b), and finally sympatry (c).

693 Phenotypic divergence may take different pathways during this cycle: gradual models predict no 694 pulse of divergence at any point in the cycle (d), whereas punctuated models involve stasis 695 punctuated by pulses, which can follow the onset of coexistence (e) or precede it (f). Note that 696 mutual exclusion in abutting (parapatric) ranges (as in $\mathbf{b}$ ) is extended when traits are similar (e), 697 and reduced when traits have already substantially diverged (f). Figure 2 | Tempo of body mass divergence for avian sister species. Stochastic pulsed models

700 provide better fits to patterns of body mass divergence and divergence time in avian sister 701 species pairs $(n=869)$, with the best fit a single pulse model ( $\triangle$ AIC relative to the multiple pulse

702 model: 797). Colors denote probability density. The probability density for any time slice

703 follows a normal distribution (most apparent in the white noise model where the probability

704 density distribution is independent of time). Relative probability density can be assessed within

705 each time slice but not across time. For clarity, the empirical data points are plotted only on the 706 white noise model. 
708 Figure 3 | Timing of body mass divergence pulses and sympatry. Comparison of timescales

709 suggests that mass divergence tends to precede sympatry among 952 avian sister species. Yellow

710 and orange lines are cumulative probability distributions of incurring a pulse under the single

711 pulse model (yellow: estimated from maximum likelihood phylogenetic tree ${ }^{36}$; orange: from 100

712 bootstrap trees). Circles are proportions of sympatric species pairs for 1-million year intervals of

713 divergence time; circle sizes represent sample sizes, numbered where $\geq 50$. For visual

714 comparison, an exponential decay model has been fitted to the proportion of sister pairs in

715 sympatry (blue curve; assumes all sympatry is secondary). If we assume that rates of transition

716 out of sympatry are low, the timing of divergence pulses is early compared to the generally

717 delayed attainment of sympatry (i.e. the yellow and orange curves are steeper than the blue

718 curve).

720 Figure 4 | Phylogenetic patterns of contact and sympatry across avian sister species.

721 Evolutionary and geographic range relationships among 952 pairs of sister species scored for

722 breeding contact (orange) and sympatry (blue), showing that both are widespread in sister

723 species across phylogeny. Pairs with breeding contact include both parapatric and sympatric

724 species pairs. The tree is derived from the Burleigh tree ${ }^{36}$, and has been pruned . Terminal

725 branches are further pruned such that tips represent the most recent common ancestors of sister

726 species pairs. There are 23 sister pairs that were scored as sympatric from range polygons, but

727 for which no breeding contact records existed in the eBird data set ${ }^{34,76}$, likely reflecting a

728 combination of sparse sampling in these sister species' ranges and limited syntopy despite broad

729 geographic overlap.

731 Figure 5 | Factors associated with the establishment of secondary contact and sympatry in

732 birds. Results of generalized linear models assessing the relative importance of predictors of

733 breeding contact (a, $\mathrm{n}=849$ sister pairs) and sympatry (b, $\mathrm{n}=440$ sister pairs with breeding co-

734 occurrence). Pairs with breeding contact include both parapatric and sympatric species pairs.

735 Relative importance is estimated as the proportion of the summed model weights for all models

736 with $\Delta \mathrm{AIC}<2$, and is indicative of the extent to which each variable is necessary to explain the 
737 variation. Pairwise interactions with relative importance $>0.6$ are indicated by the numbers 738 within the bar for each variable. 

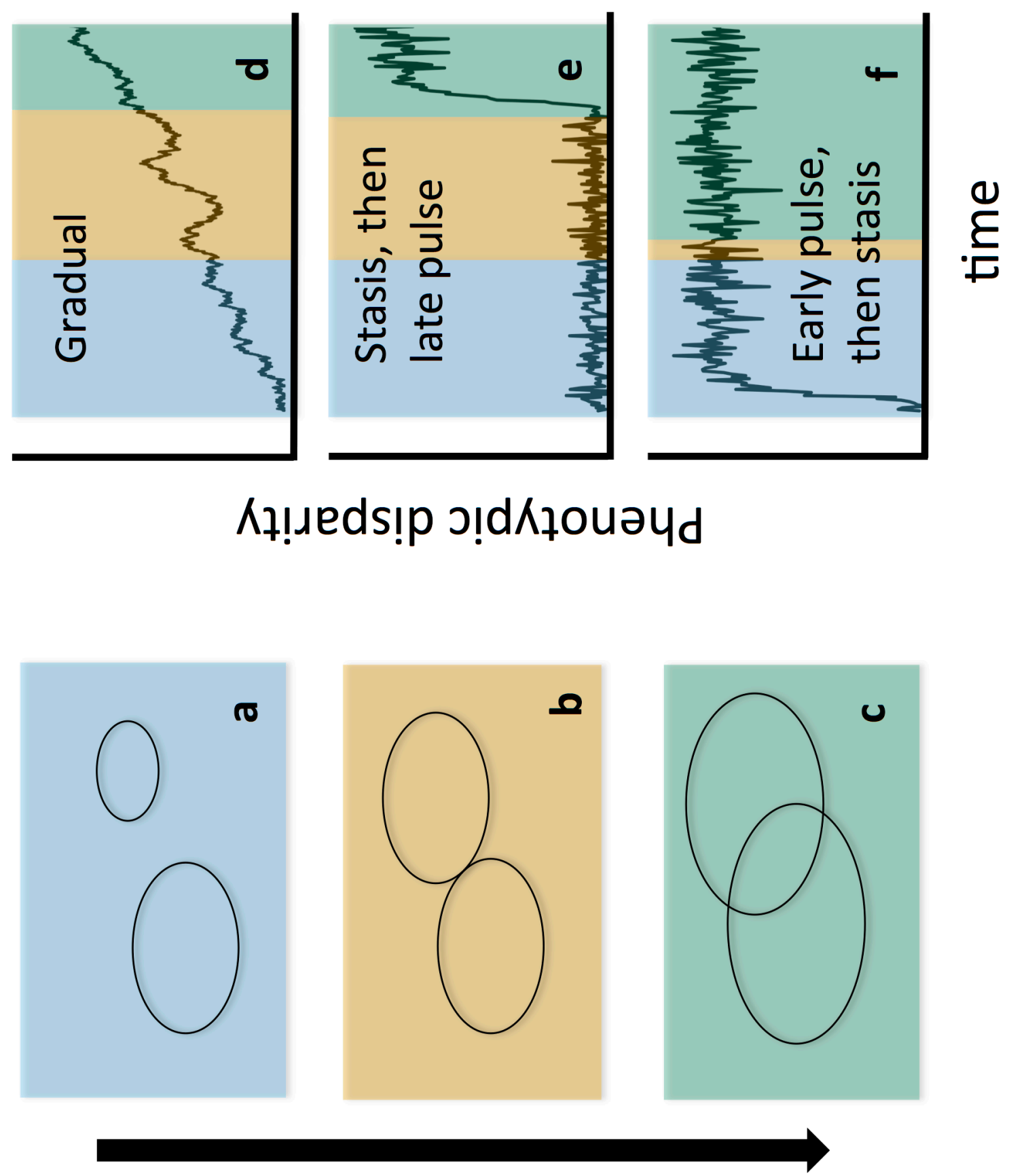

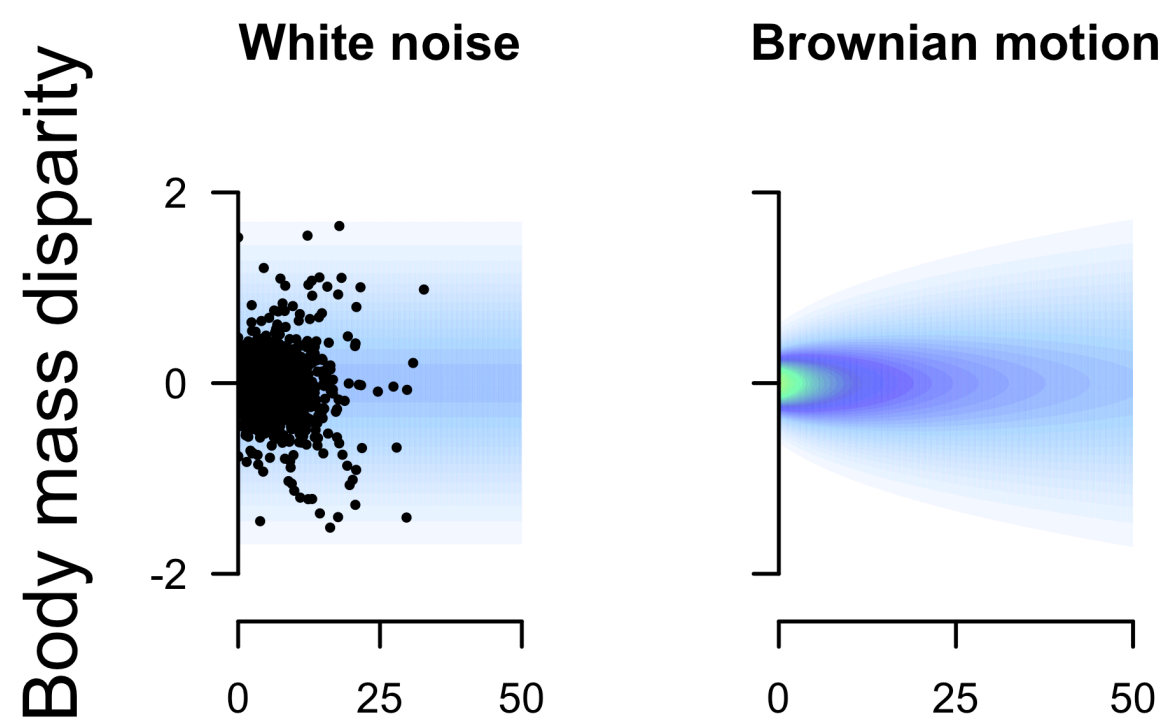

Single pulse

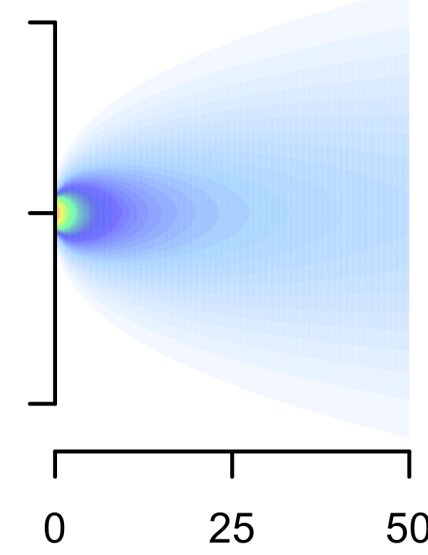

Divergence time (My)
Multiple pulse
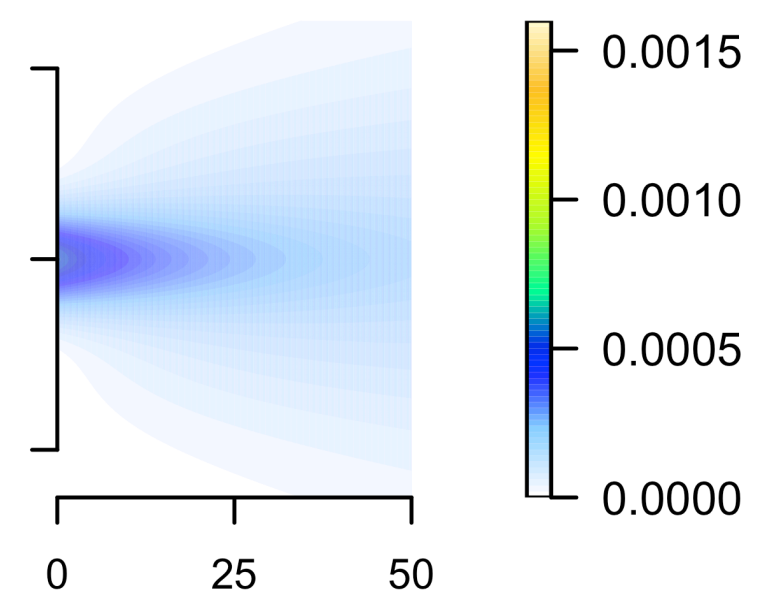


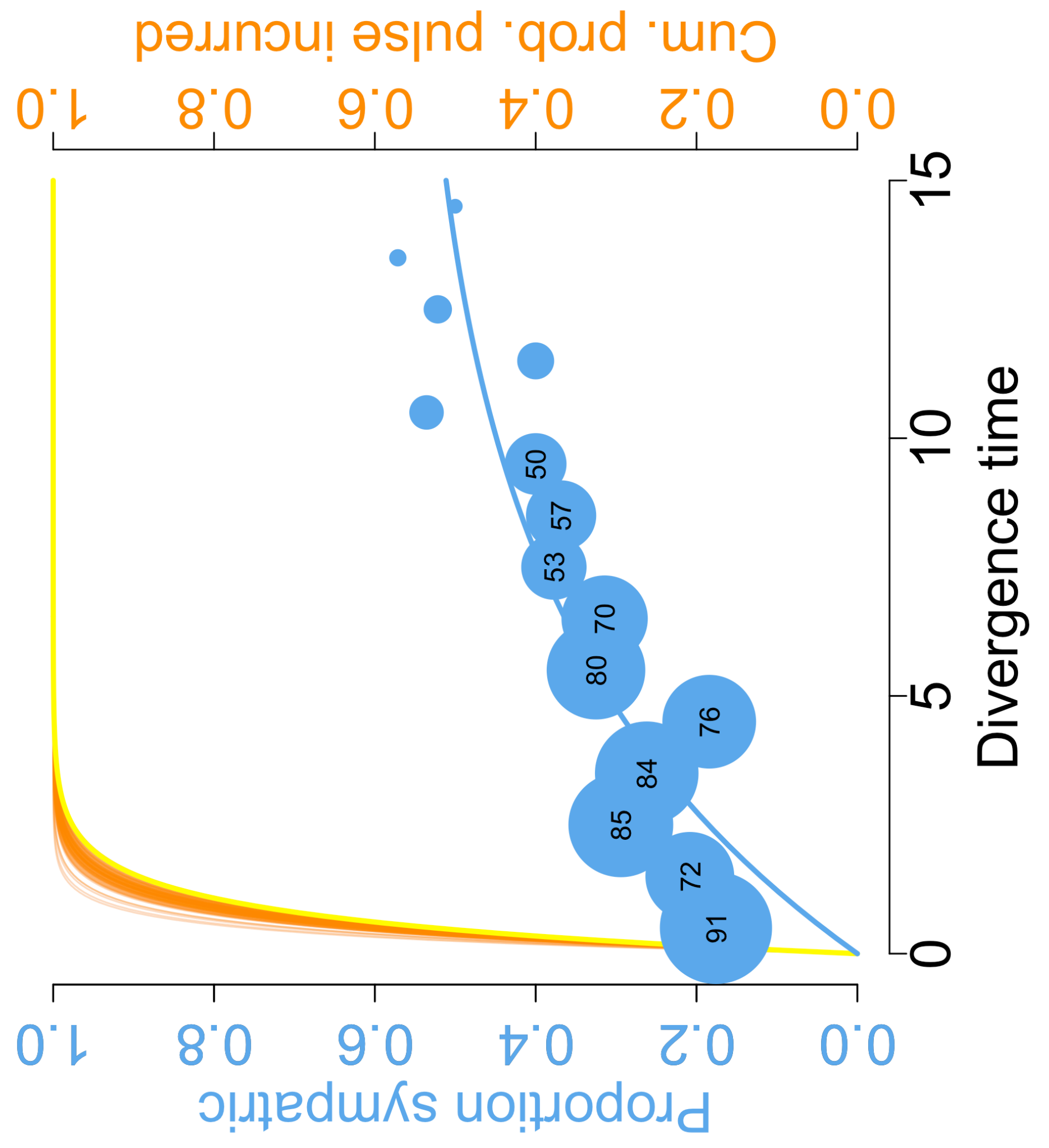







Secondary contact

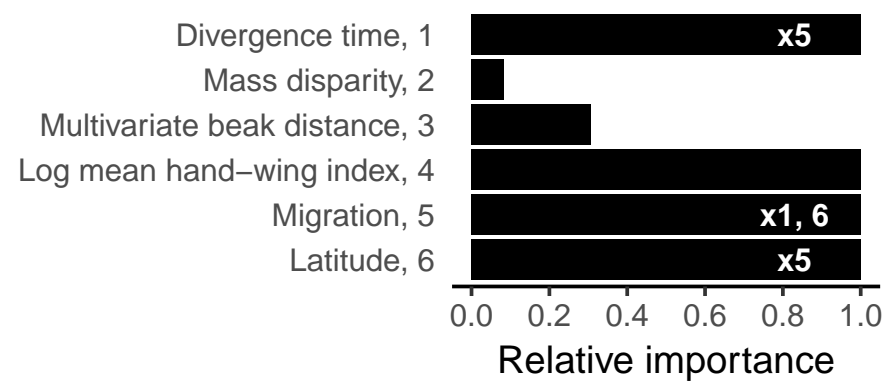

Sympatry

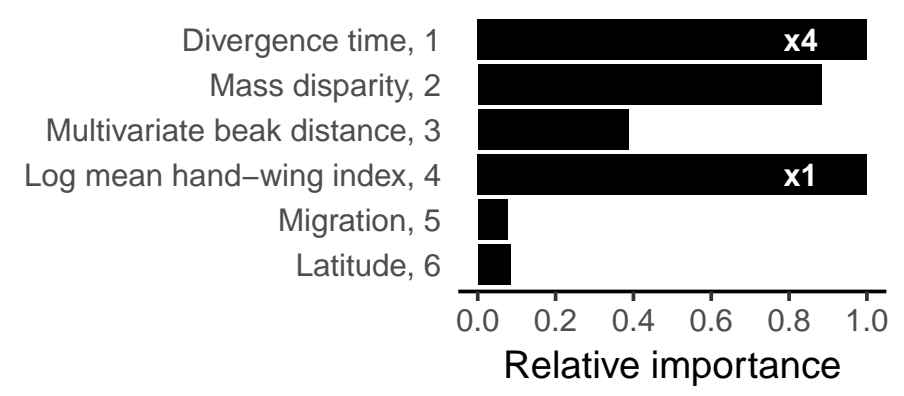

